\title{
15
}

\section{Interactive Voice Response - More Power to the Students}

\author{
Graeme Burton \\ Student Secretariat, Griffith University, Australia, 4111
}

\begin{abstract}
Interactive Voice Response Technology allows access to institutional computer systems by way of a telephone. This means that students can be given access to their own information at any time from virtually anywhere they have access to a telephone. They can find out what it is and change it if they want. Because the host computer system still applies course rules, the changes made by students can still fall within the requirements of the institution.
\end{abstract}

Keyword codes: H.5.2; I.2.1; C.2.4

Keywords: User Interfaces; Applications and Expert Systems; Distributed Systems

\section{WHAT IS VOICE RESPONSE?}

Voice Response should not be confused with Voice Recognition. Voice Response is concerned with information that is produced by a computer and presented to a caller - Voice Recognition is concerned with information going the other way.

The voice presented to the caller by the Voice Responses System (VRS) can be in one of two forms - synthesised or digitised. Synthesised voice is machine produced and is reminiscent of 'talking robots'. Although text-to-voice processing (having a computer translate a word of text into sounds) is improving all the time, the resultant voice still sounds artificial, and algorithms to cope with unusual pronunciations or different pronunciations of the same word still have a fair way to go. Digitised voice, on the other hand, is voice that has been recorded by someone. Consequently, it sounds like a person talking on the other end of the telephone. The quality and presentability are much higher than synthesised voice. It does, however, mean that every word to be spoken must be recorded.

A Voice Processing System (VPS) goes one step further - it allows you to record parts of messages and it combines the appropriate parts into a complete message and speaks it to the caller. For example, you might record the following messages:

\section{Your enrolment for semester \\ is as follows \\ the digits 1 to 9}

Depending on the value of the semester required, the VPS can put the appropriate parts together and produce a single message such as "Your enrolment for semester two is as follows" and present it to the caller. This is a very powerful feature of Voice Processing Systems. 


\section{WHAT IS INTERACTIVE VOICE RESPONSE?}

Interactive Voice Response (IVR) Systems allow two-way interaction - the caller can give the system information as well as receive it. This information can be used to navigate around the options available on the VPS or as input into a remote database. Students, for example, can enter information that writes to or reads from a remote host database. The VPS may ask for a student number. When the caller enters it, the VPS sends it to the host computer for validation. The student's enrolment information might then be sent back to the VPS to be spoken to the student.

\section{HOW IS INFORMATION ENTERED AT THE TELEPHONE?}

There are three ways to enter information at the telephone:

Voice Input.This is Voice Recognition. The caller speaks into the telephone handset and the VPS interprets what is said and translates it into data that can be sent to a computer. At the moment, Voice Recognition is in its infancy. The digits 0 to 9 , yes and no are about all they can understand. There are also problems understanding people with pronounced accents, heavy colds, etc. It is very slow and rather unreliable, but it is undoubtedly the way of the future.

Pulse Input. This is the way rotary dial telephones (and many of the earlier push button 'phones) work. An electronic tone is not produced - rather, you can hear a series of mechanical clicks - a different number for each digit. Push button 'phones that are decatic (use pulses) produce the same sound. Many Voice Processing Systems can detect pulses and interpret them, but they are not $100 \%$ reliable.

Tone input. Modern telephones use electronic tones to send information. As you dial a number, you will hear a tone for each number pressed. This method of sending information to a VPS is by far the most reliable. All new Telecom 'phones are of this type as are new public telephones and almost all of those available in stores. Many 'phones have a switch that allows you to use either tone or pulse dialling. Tone input is much faster than either of the other two methods and much more reliable. Touchtone 'phones also give you the advantage of two extra keys - the \# and the *. These can be used to advantage during interaction with a VPS, particularly to indicate that input is complete, to have a prompt repeated or to correct typing errors. Interrupting a message being spoken and typing ahead is really only available with tone input. This feature allows the caller who is familiar with the system to ring, get immediately to the option they want, do what they want to do and finish the call - all in a minimum time and for minimum cost.

\section{HOW DOES IT WORK?}

Interactive Voice Response typically involves the following steps:

- The caller rings the VPS

- The VPS speaks messages and give the caller a list of options

- The caller enters the required option

- The VPS asks for information

- The caller provides it

- The VPS interprets it, decides what has to be sent to the host computer and sends it 
- The host computer takes the data provided, does what it has to with it and sends data back to the VPS

- The VPS interprets the data received from the host computer and speaks information to the caller.

The interaction is very similar to using a terminal on the host computer system. The keypad on the telephone replaces the keyboard on the terminal and the spoken voice in the telephone earpiece replaces the text on the screen of the terminal. The VPS handles the necessary translation in between.

\section{WHAT TELEPHONE ACCESS IS REQUIRED?}

The VPS can be connected to the telephone system in any of a number of ways, depending on the service you wish to provide to your clients - your students.

- Normal 'phone access works fine for students who live in the local area, but can be very expensive for those who may wish to use the service from a distance.

- 008 service is fair on all students but means that the institution pays all the costs of calls.

- 0055 will cost the caller according to the length of the call, but the charge will be the same per minute from anywhere in Australia. The cost can provide an incentive for efficient use of the system, thus avoiding clogging by callers who are 'wasting time' on the system. It can also provide revenue to help defray the costs of maintaining the system.

- Direct Indial (DID) can be used with any of the above systems and can pass the last few digits of the number called through to the VPS - useful in a shared system so the VPS knows which application to connect the caller to.

- There are also other methods of access through the telephone system - Telecom can provide advice on them.

\section{HOW BIG DOES THE SYSTEM NEED TO BE?}

The size of the VPS required will depend on a number of factors. You will need to choose a critical period when the system will be heavily used and, for this period, estimate things such as:

- the number of students who will use it (ns)

- the average number of calls per student expected (nc)

- the average duration of a call in minutes (cd)

- the number of hours per day that the system will be available (hd)

- the number of days in this period that the VPS will be available (d)

- whether or not a call scheduling system will be used?

- whether or not the system is shared or dedicated?

The system size can be estimated as follows:
usage (in hours)
$=(\mathrm{ns} \times \mathrm{nc} \times \mathrm{cd}) / 60$
availability (in hours)
Number of lines
$=\mathrm{d} x$ hd
$=$ usage $/$ availability 
For example, in a 3 week enrolment period:

A group of institutions with a total of 150000 students (ns)

Average calls expected per student is 3 (nc)

Average duration of each call expected to be $4 \mathrm{~min}$ (cd)

Hours per day the system available is 18 (hd)

Days in the period the system available is 21 (d)

$\begin{array}{lll}\text { Usage } & =(150000 * 3 * 4) / 60 \text { hours } & =30000 \\ \text { Availability } & =21 * 18 \text { hours } & =378 \\ \text { Required lines } & =\mathbf{3 0} 000 / 378 & \end{array}$

\section{WHAT ARE ITS USES?}

The uses of Interactive Voice Response in a Tertiary Institution are limited only by the imagination. Some examples are:

- an information or help line

- to inquire about enrolment or preference details

- to change enrolment or preferences

- to follow the progress of a tertiary application

- to accept or reject an offer

- to enrol or re-enrol

- to cancel enrolment

- to notify leave of absence

- to pay fees and charges

- to find results

- to order an academic transcript

- to find a personal exam timetable

- to apply to graduate or provide graduation information

- to vote in elections

\section{WHAT ARE ITS LIMITATIONS?}

The limitations of the current IVR technology are:

- You will need a touchtone 'phone for most effective use

- There are problems in entering alphabetic characters - codes can be used but these are clumsy and slow - it is probably better and certainly easier to stick with strictly numeric codes, even if this means having a set of codes just for IVR use.

- You will need to accommodate hearing impaired students - this is probably best done by providing a service at your institution for these people - have someone on hand to help them.

\section{WHAT ARE ITS ADVANTAGES?}

The advantages of IVR are significant and are the reasons that the relatively large upfront capital cost to the institution of installing IVR equipment is well and truly worth it. Some of the more obvious advantages are:

Your student data is more reliable and timely - students have access to their own information and can ensure that it is correct. They are the ones who have the most reason to ensure it is accurate. Once they authorise a change to be made, it is 
done - there is no delay waiting for forms to be processed (or misplaced). In critical enrolment periods, all the data is on the system at the deadline - there is no delay waiting for forms submitted at the last minute to be processed.

Students are responsible for the accuracy of their own enrolment - no longer is there the I submitted a change form and we did not receive it debate. The student has access to their information and the ability to get it right (within the normal rules). The responsibility has been moved from the administrators to the students.

Payment of fees and charges can involve a direct funds transfer - once the system tells the caller that the payment has been accepted, the institution actually has the money. This obviates the following up of dishonoured cheques and payments that cannot be identified.

Students know changes have been made - they no longer have to submit a piece of paper and hope that it gets processed. Once the IVR system says that the change has been made, it has. They can check at any time to ensure that things are as they should be.

Problems are picked up early and the student knows about them. If a student tries to enrol in a course that is not offered, or enters an incorrect course code, the system will tell them on the spot and disallow the operation. They will know that there is a problem immediately and can follow it up straight away.

IVR is eminently convenient - students can have access to the institution's computer system from home, the car, the beach, the pictures - in fact from almost anywhere - and almost anytime (on our system, 20 hours a day 7 days a week). Most transactions can be concluded in the ads during the movie or the football.

IVR is cheap - even on a 0055 number, doing changes over the telephone is probably cheaper than getting in the car or a bus and travelling to the Institution and back.

Savings in casual labour. The savings in data entry time can be enormous, as can the savings in time involved in chasing up errors made on forms by students - they will know their enrolment is not correct when they tried to enter the error and will have a vested interest in following it up to ensure that things are put right.

\section{SUMMARY}

Interactive Voice Response Technology is the way Tertiary education administration must move. It has the potential to radically alter the way we administer our clients. From the institutional point of view, it will result in significant cost savings as well as allowing us to work with data that is more reliable, timely and up to date. From the students point of view, it will give them ready, convenient and reliable access to their own enrolment information, as well as the ability to fix it if it is wrong. It will put the responsibility for a student's enrolment squarely on the shoulders of the student and give them the ability to manage it within the normal administration rules of the institution. It will give more power to the students - the power to control their own enrolment and get it right - something we are often blamed for being unable to do. 